\title{
The Use of Micro-Electro-Mechanical Systems (MEMS) in the Construction Industry
}

\author{
Amaury A. Caballero and Kang K. Yen \\ Department of Construction Management caballer@fiu.edu \\ Department of Electrical and Computer Engineering yen@fiu.edu \\ Florida International University \\ Miami, Florida, USA
}

\begin{abstract}
Micro-electro-mechanical systems (MEMS) have been identified as one of the most promising technologies and will continue to revolutionize the industry as well as the industrial and consumer products by combining silicon-based microelectronics with micro-machining technology. All the spheres of industrial application including robots conception and development will be impacted by this new technology. If semiconductor microfabrication was contemplated to be the first micro-manufacturing revolution, MEMS is the second revolution. The paper reflects the results of a study about the state of the art of this technology and its future influence in the development of the construction industry. The addressed questions are: technical and economical effects of MEMS application in the industry and analysis of their use in Japan, Europe, and the USA. Also several examples of MEMS applications in construction are presented.
\end{abstract}

KEY WORDS: MEMS, Electronics, Construction, Sensors, Automation

\section{INTRODUCTION}

The integration of mechanical elements, sensors, actuators, and electronics on a common silicon substrate through micro-fabrication technology leads to what is known as micro-electro-mechanical systems (MEMS). These devices permit unprecedented levels of functionality, reliability, portability, and ruggedness at low prices. Their properties open new possibilities for the use in any branch of technology, including any construction process.

The integration of mechanical elements, sensors, actuators, and electronics on a common silicon substrate through micro-fabrication technology leads to what is known as micro-electro-mechanical systems (MEMS). These devices permit unprecedented levels of functionality, reliability, portability, and ruggedness at low prices. Their properties open new possibilities for the use in any branch of technology, including any construction process.

MEMS are small, integrated devices or systems that combine electrical and mechanical components. They range in size from the sub micrometer (or sub micron) level to the millimeter level, and there can be any number, from a few to millions, in a particular system. These systems can sense, control, and activate mechanical processes on the micro scale, and function individually or in arrays to generate effects on the macro scale. They are a fabrication approach that conveys the advantages of miniaturization, multiple components, and microelectronics to the design and construction of integrated electromechanical systems [Vittorio 2001].

The main advantages obtained with this technology can be condensed as: Reliability, Intelligent Products, Inexpensive Production, and Clean Production.

The paper reflects the results of a study about the state of the art of this technology and its influence in the future development of the construction industry. The addressed questions are:

- MEMS application in the construction industry

- Analysis of their use in Japan, Europe, and the USA 


\section{FABRICATION TECHNOLOGIES AND APPLICATIONS.}

MEMS are built in ways similar to the way the integrated circuit is built. By pattering various layers of polysilicon as they are deposited, and releasing part of the structures, the devices are capable of motion. The MEMS industry has employed three distinct processing technologies, more or less defined by the height of the finished structure [Singer 2002]: bulk micro-machining, surface micro-machining, and a process commonly known as $L I G A$, an acronym from the German words for lithography (lithografie), electroplating (galvanoformung) and molding (abformung). For most of the highest-volume applications, there has been a tendency to use surface micromachining, not only because it makes it easier to integrate circuitry with the device, but also makes it possible to use the same equipment developed for IC manufacturing.

MEMS devices have a wide range of application, covering from medicine, computers and communication to manufacturing systems. Some of them are:

- Micro-robots

- Gyroscopes

- Micro-optical systems for fiber-optics communications

- Switches, varactors, inductors, and resonators

- Micro-tweezers

- Neural probes

- Accelerometers

- Pressure Sensors

- Micro-engines

\subsection{Pressure Sensors}

Pressure sensors normally have a flexible diaphragm that deforms in the presence of pressure difference. The deformation is converted in an electrical signal that appears at the sensor output.
The MEMS pressure sensors frequently use the piezoresistive effect. Due to this, the change in electrical resistivity that occurs with application of mechanical stress produces changes in electrical variables at the device's output. A more detailed description can be found in [Silicon Microstructures, Inc].

On figure 1 it is shown the fraction of the total that corresponded to accelerometers, gyroscopes, and pressure sensors in US in the year 2001 and the expected value in 2008 [Carrillo].

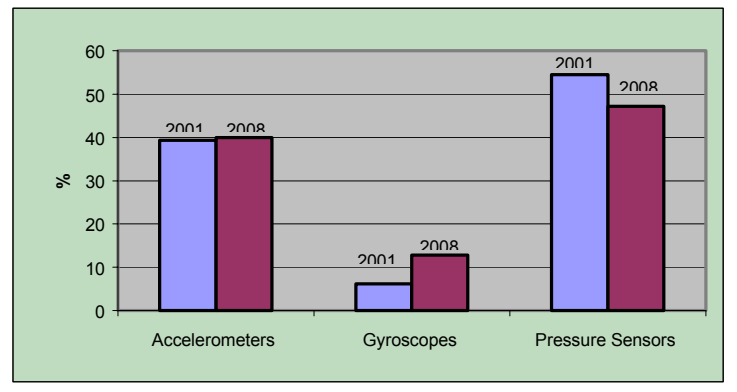

Figure 1. Total MEMS Sensor Market in US

\subsection{Pavement condition monitoring}

A very interesting application is presented by AttohOkine [Atto-Okine 2002]. The analysis of the use of MEMS for collecting data reflecting the conditions of the pavement is performed. The idea is based on the introduction of microsensors in the asphalt to monitor parameters critical to the safe operation and performance. The system consists of a network of sensors embedded into the pavement structure. The collected data is transmitted via low power radio link to a receiver and data logger located on the side of the road. As was reflected in the previous cited paper, several issues have to be considered when applying this technology for pavement condition monitoring. Among them are cited: the effect of asphalt medium on MEMS, where these devices will be embedded in the pavement, how long will last the devices, and how much will cost this method of collecting data.

\subsection{Flaw detection in structures}

Many devices have been developed for flaw detection, using laser, fiber optics, etc. Ultrasonic flaw detectors are also used, based in the Doppler principle. When using ultrasonic detectors, several variables affect the ability of ultrasound to locate defects. These include the frequency of ultrasound 
impulses, pulse length, type and voltage applied to the crystal, properties of the crystal, backing material, transducer diameter, and the receiver circuitry of the instrument. Among the different options for detecting flaws in metallic structures, the use of capacitive sensors [Jain 2002] appears like a very interesting one. The device uses a capacitive MEMS transducer, where a diaphragm deflection produces a change in the capacitance that can be detected electrically. The authors have developed a resident ultrasonic flaw detection system to be mounted at critical locations on metal structures. The device is polled remotely using RF communication. The chip is approximately $1-\mathrm{cm}$ square and contains 23 detectors. In the tests commercial ultrasonic transducers with operating frequencies of $3.5 \mathrm{MHz}$ and $5 \mathrm{MHz}$ were the signal sources.

\subsection{Bulk and Surface Acoustic Wave Sensors}

Using piezoelectric MEMS as transducers of different physical properties employing silicon offer many advantages. Ivanov analyzes the characteristics of advanced sensors for detecting simultaneously various parameters such as temperature, pressure, electric and magnetic fields, etc [Ivanov 2000]. These sensors provide not only high signal-to-noise ratio in a wide dynamic range but also well cross-sensitivity.

Bulk and surface acoustic wave resonators have been used extensively in the design of multifunctional physical and chemical sensors. Because frequency may be measured with higher accuracy than any other parameter, mechanical resonators are well suited for the design of highsensitivity sensors. A resonator without any load film, in vacuum behaves like an unbounded, stressfree plate. If the resonator is loaded with a thin film, the boundary conditions will be modified. A dielectric film modifies mechanical boundary conditions, while a conducting film modifies both mechanical and electrical boundary conditions. Mechanical and electrical perturbations cause resonant-frequency shifts. The regime of oscillation of a piezoelectric resonator can be modified by mechanical or electrical perturbations originating from the surrounding medium. As indicated by Ivanov, "when a free quartz resonator is brought in contact with a solid or fluid medium, some acoustic energy is drained out of the resonator, resulting in damping of the oscillations. The acoustic coupling with the loading medium and the amount of acoustic energy returned back to the resonator defines the resonance frequency shift. Loading a quartz resonator may result in a decrease of the Q-factor, a resonance frequency shift or both effects at the same time".

This property can be used for creating MEMS devices which, embedded in the building structure, can detect and store information related to fatigue or any change in the medium characteristics.

\section{DEVELOPMENT OF MEMS TECHNOLOGY IN USA, EUROPE, AND JAPAN}

MEMS devices have successfully established highvolume commercial markets including accelerometers and pressure sensors for automotive applications, inkjet print heads, and digital micromirrors for image projection. In addition to major industry players, there exist many research institutes, government and private laboratories, and universities all around the world, pursuing researches related to this technology. A geographical breakdown on MEMS fabrication is given in Figure 2 [Yole 2003]. As can be seen, from this data, the US occupy the first place, followed by the European countries and in third place, Japan.

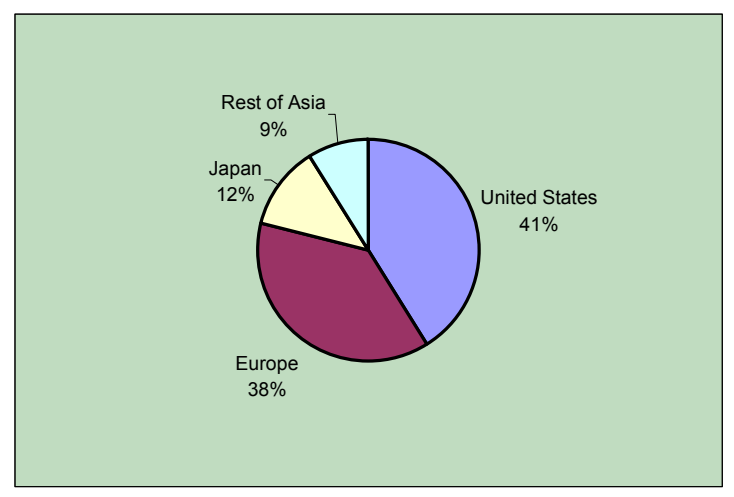

Figure 2. Geographical Breakdown of MEMS Fabrication

Data published from a study of the NEXUS Task Force [MCC/WTEC 2000] show that in Europe "the automotive sector was the market driver in the first phase of MEMS commercialization, but has been supplanted by the IT peripherals sector (including 
HDD read/write heads and inkjet print heads)". Biomedical, telecommunications, mass storage, and display applications are increasing their volume. The device classes being pursued mostly, as per [WTEC Hyper-Librarian], fall into the following categories: Fluidic MEMS, Mechanical Transducers, Optical MEMS, and Electrical MEMS Switches.

Japanese programs have been significant in the development of sensors, actuators, and MEMS in the past through programs at a number of universities and companies, and the leadership of Japanese industry in consumer products puts them in an excellent position to benefit from MEMS technology.

During year 2000, the MEMS industry in USA was estimated from 2 to 5 billion dollars, and in 2004 should grow up in the order of 12 to 15 billion dollars. It is expected that in 2004 there will be 5 MEMS devices per person in the country. In year 2001, 163 companies were established with a total of 6000 employees [Yole 2003]. This numbers have been growing every year.

\section{SOME CHALLENGES}

Packaging MEMS-based sensors is the critical issue that has and will have a strong impact in the North American MEMS-based sensors market. This challenge is crucial in industries such as the medical and process control. Consequently, MEMS sensor manufacturers need to build their own packaging, which translates into high manufacturing costs as opposed to the standard integrated circuit (IC) packaging technology, which tends to be more costeffective when mass-producing sensing devices. The packaging of MEMS-based sensors is probably the greatest challenge facing the MEMS industry [MEMS 2002].

MEMS are too small for traditional batteries. These batteries must fit inside devices smaller than the width of a human hair and provide long-lasting power. A way of solving this problem is proposed by Bruce Dunn. He proposes changing from twodimensional sheets of electrodes to rods arranged in a three-dimensional array in which hundreds of rods are stacked next to each other like tubes on a flatbed truck. Each rod is only a thousandth of a centimeter in size. The group is currently designing a battery five millimeters in size, which presents significant design challenges [UCLA 2002].

\section{CONCLUSIONS}

The development of MEMS is demanding higher levels of electrical-mechanical interaction, as well as a higher level of knowledge of the physical world. Their use increases the systems' properties like reliability and level of integration. The development of microdevices in which are embedded the electronic circuits, sensors, actuators and engines, open new ways of solving industrial problems at lower cost and increased quality.

MEMS sensors utilization permit to avoid the necessity of point-to-point wiring, realizing a digital output format, and obtaining greater precision. Embedded sensors in different type of structures permits the creation of what is called "smart structures", which can be used in civil and mechanical engineering projects.

The US is the country realizing more investments in this direction, followed by the European community and Japan. The major field of application of MEMS in industry is in first place different type of sensors. Also the wireless MEMS because they offer the possibility of achieving significantly lower power consumption and more compact integration.

There are challenges to face in the introduction of MEMS in the market. Among them, the packing, small and long-lasting batteries, and their behavior under different environmental conditions. 


\section{REFERENCES}

[Vittorio 2001]. Vittorio, Salvatore A.. MicroElectromechanical Systems (MEMS), Cambridge scientific abstracts. October 2001. www.csa.com/hottopics/mems/oview.html

[Singer 2002]. Singer, Peter. MEMS Diversity and Advance. Semiconductor International. October $1^{\text {st }}$, 2002.

[Silicon Microstructures, Inc]. Silicon Microstructures, Inc. Simplified Description in the Process to Fabricate Piezoresistive Pressure Sensors.www.si-

micro.com/pdffiles/PressureFab.pdf.

[Carrillo]. Carrillo, Daniela. MEMS Sensor Technology: Present and Future. Frost.com.. www.frost.com/prod/news.nsf/0/2C5351EB5F6D41 4A86256CBE005C5ED4

[Atto-Okine 2002], Attoh-Okine Nii O. MEMS Application in Pavement Condition MonitoringChallenges. Proceedings of the $19^{\text {th }}$ International Symposium on Automation and Robotics in Construction (ISARC) 2002, pp. 387-392. Gaithersburg, USA.

[Jain 2002]. Jain, Akash, D.W. Greve, and J. Oppenheim. A MEMS transducer for Ultrasonic Flaw Detection. Proceedings of the $19^{\text {th }}$ International Symposium on Automation and Robotics in Construction (ISARC) 2002, pp. 375380. Gaithersburg, USA.

[Ivanov 2000]. Ivanov D. V. Advanced Sensors for Multifunctional Applications. $\underline{\text { JOM-e }}, \underline{\mathbf{5 2}}$ (10) $\underline{\mathbf{2 0 0 0}}$,

[Yole 2003]. Yole Développement. Overview of the European MEMS Industry. Lyon, France, 2003. www.yole.fr.

[MCC/WTEC 2000]. MCC/WTEC Strategic Technology Tour Report on MEMS and Microsystems in Europe. January 2000. www.wtec.org/loyola/mcc/mems eu.

[WTEC Hyper-Librarian]. WTEC HyperLibrarian. The State of the Art in Europe.
Published: January 2000; www.wtec.org/loyola/mcc/mems_eu/Pages/Ch apter-1.html.

[MEMS 2002]. MEMS Revolutionizing the Sensors Industry. Instrumentation and control. www.etinstrumentation.com/oct nov2002/analytics b.html.

[UCLA 2002]. UCLA Design could power MEMS Devices. Small Times Media 2002. 